\title{
DIABETES
}

\section{Early initiation of intensive therapy might benefit children with type 2 diabetes mellitus}

The results of a 6-year, phase III, randomized study published in The New England Journal of Medicine suggest that many children with type 2 diabetes mellitus (T2DM) need more than one drug to achieve good glycaemic control. "Metformin monotherapy was not as effective in maintaining glycaemic control in the kids as we expected from adult studies or from the investigators' clinical experience," comments Phil Zeitler, chair of the TODAY study group.

\section{We view our findings as} a proof of principle for using more intensive pharmacologic therapy... 77

The trial was designed to investigate the influence of puberty on the course of T2DM and to assess the possible benefits of intensive glycaemic control during puberty (a time of increased insulin resistance). The participants were 699 children with T2DM aged 10-17 years, from 15 centres in the USA. All initially received metformin monotherapy (1 $\mathrm{g}$ twice daily) to attain $\mathrm{HbA}_{1 \mathrm{c}}$ levels $<8 \%$. The children were then randomly allocated to one of three therapeutic approaches: continued metformin monotherapy, metformin plus a second drug, or metformin plus an intensive lifestyle intervention.

Half of the children were not able to maintain $\mathrm{HbA}_{1 \mathrm{c}}$ levels $<8 \%$ while receiving metformin alone. "However, half of the kids did do well on metformin monotherapy and look like they have a long-term response," notes Zeitler. Intriguingly, however, single-agent metformin was particularly ineffective in African American participants, and girls responded better than boys to addition of a second agent, although Zeitler stresses that these prespecified subgroup analyses involved small numbers of patients and did not take into account potential confounding variables.

"Ideally, it would be nice to be able to pick out from the beginning those who will respond to relatively minimal intervention (improving as they get out of puberty, much like women with gestational diabetes) and those who have a more rapidly progressing form of T2DM that requires more intensive therapy," he adds. "There may be subsets of kids who are easier or harder to treat."

The second agent used in this study was rosiglitazone, however-which is no longer a realistic option in light of concerns over its possible cardiovascular adverse effects. "We view our findings as a proof of principle for using more intensive pharmacologic therapy in kids at diagnosis, not as a specific indication for use of rosiglitazone," comments Zeitler. Comparative studies will be needed to determine the optimal combination therapy in children who do not respond to metformin. The children who received intensive lifestyle modification showed increased weight loss, says Zeitler, but the change was fairly small and did not

translate into improved glycaemic control. "It's important that this result is not interpreted as meaning that no lifestyle intervention is important," stresses Zeitler. "All the kids in the study received routine dietary counseling and encouragement to exercise and we have soon to be published data showing that this approach was indeed effective. But the very intensive intervention we did was not additionally beneficial." And the key message? "We need to intervene earlier as kids begin to lose glycaemic control, and not necessarily assume they aren't taking their medications," concludes Zeitler.

\section{Caroline Barranco}

Original article TODAY study group. A clinical trial to maintain glycemic control in youth with type 2 diabetes. N. Engl. J. Med. doi:10.1056/NEJMoa1109333 